and others have called for reduced eponym coinage and use in favor of descriptive terms [3].

Eponyms commonly recognize one or two people, which may reflect influence, chance, seniority, politics, gender, or language rather than bearing witness to global discourse and collaborative scientific inquiry. For example, Tsuya Sakurai described the melanocytic iris hamartomas characteristic of neurofibromatosis type 1 [4], accompanied by her detailed illustrations, two years before Lisch's paper was published in the German language literature. Syndromes, the subject of our article, are particularly likely to be identified through the work of multiple individuals, each of whom describes clusters of signs and symptoms that may be subsequently identified as syndromic.

In the current era, an additional concern with eponyms is that varying use of the possessive and non-possessive forms is a challenge for search engines, which may produce incomplete disease-specific results depending on the term used [5]. This is a hindrance to scholarly research, medical writing, and information dissemination for patients and clinicians alike.

Eponyms honor contributors, serve as memory tools, and may be simpler than names routed in genetics, function, or symptoms (consider the eponymous lysosomal storage disorders). We agree with Thomas and Gunasekera that the decline in eponyms is not "precipitous," and there is no need to rapidly expunge eponyms from use. However, the empirical decline in eponym coinage as well as many authors' and investigators' reticence to eponyms suggests that future syndromic eponyms attributed to both male and female ophthalmologists will be rare. Thus, we recognize the historical contributions of the few women for whom ophthalmic syndromes are named.

\section{Compliance with ethical standards}

Conflict of interest The authors declare that they have no conflict of interest.

Publisher's note: Springer Nature remains neutral with regard to jurisdictional claims in published maps and institutional affiliations.

\section{References}

1. Van Tassel SH, Segal KL, Ciralsky JB, Sun G. Eponymous women in ophthalmology: syndromes with prominent eye manifestations named after female physicians. Eye Lond Engl. 2018;32:1293-5.

2. Thomas PB. Are medical eponyms really dying out? A study of their usage in the historical biomedical literature. J R Coll Physicians Edinb. 2016;46:295-9.

3. Cogan DG. The rise and fall of eponyms. Arch Ophthalmol. 1978;96:2202-3.

4. Sakurai T. Multiple neurofibroma patient showing multiple flecks on the anterior surface of the iris. Acta Soc Ophthalmol Jpn. 1935;39:87-93.

5. Ayesu K, Nguyen B, Harris S, Carlan S. The case for consistent use of medical eponyms by eliminating possessive forms. J Med Libr Assoc Jmla. 2018;106:127-9.

\title{
Comment on "Vitrectomy with scleral buckling versus with inferior retinectomy in treating primary rhegmatogenous retinal detachment with PVR and inferior breaks"
}

\author{
Upma Awasthi $^{1} \cdot$ Rohini Grover $^{1} \cdot$ Chetan Videkar $^{1} \cdot$ Abhishek Varshney $^{1}$ \\ Received: 21 November 2018 / Accepted: 29 November 2018 / Published online: 18 December 2018 \\ (c) The Royal College of Ophthalmologists 2018
}

Upma Awasthi

upma.awasthi2@gmail.com

1 Department of Vitreoretina, CL Gupta Eye Institute, Moradabad 244001 Uttar Pradesh, India
To the Editor

We read the article by Eleinen and Mohalhal [1] with great interest. We applaud them to do head on comparison of scleral bucklng (SB) and retinectomy (RR) as a primary approach in patients with rhegmatogenous retinal detachment (RRD) with proliferative vitreoretinopathy (PVR) with inferior breaks. However, we would like to comment upon few points. 
In phakic patients, cataract surgery was done in the same sitting prior to RD surgery. Authors should have mentioned the method of IOL power calculation. In our experience it is better to pass the buckle before phacoemulsification and intraocular lens (IOL) implantation as maneuvering during buckling may cause anterior chamber instability and IOL decentration. Surgeries were done by multiple surgeons. Different surgeons have different approach for a case so it would have affected the decision for preferring one surgical technique over the other. Also, it would have been more informative if the criteria for preferring surgical technique would have been mentioned.

Authors have preferred 5000cS silicon oil in all cases with oil removal at 3 months. High viscosity silicon oils are preferred in cases where long term or permanent tamponade is required [2]. Moreover, 5000cS oil is lighter than water, thus inflammatory cytokines get settled in inferior unsupported space leading to inferior PVR changes [3]. So, if inferior tamponade was the purpose of preferring $5000 \mathrm{cS}$, then heavy oils would have been better choice [4].

Authors have done regression analysis for finding correlation of seven factors with recurrence of RD. In multivariate regression, testing too many variables for the small sample size will overestimate associations.

Causes of higher IOP and better visual acuity in buckle group should have been discussed. Subgroup analysis for the grades of PVR would have been more informative regarding dealing with severe PVR. We appreciate authors for their choice of doing photocoagulation of bare choroid in cases of RR. Once again we applaud authors for sharing their experience.

\section{Compliance with ethical standards}

Conflict of interest The authors declare that they have no conflict of interest.

Publisher's note: Springer Nature remains neutral with regard to jurisdictional claims in published maps and institutional affiliations.

\section{References}

1. Eleinen KG, Mohalhal AA, Ghalwash DA et al. Vitrectomy with scleral buckling versus with inferior retinectomy in treating primary rhegmatogenous retinal detachment with PVR and inferior breaks. Eye. 2018.16:1.

2. Barca F, Caporossi T, Rizzo S. Silicone oil: different physical proprieties and clinical applications. BioMed research international. 2014;2014:502143.

3. de Juan E, Jr, McCuen B, Tiedeman J. Intraocular tamponade and surface tension. Surv Ophthalmol. 1985;30(1):47-51.

4. Joussen AM, Rizzo S, Kirchhof B et al. Heavy silicone oil versus standard silicone oil in as vitreous tamponade in inferior PVR (HSO Study): interim analysis. Acta Ophthalmol. 2011;89:e483-9.

\title{
Vitrectomy with scleral buckling versus with inferior retinectomy in treating primary rhegmatogenous retinal detachment with PVR and inferior breaks
}

\author{
Khaled G. Abu Eleinen ${ }^{1}{ }^{1} \cdot$ Ahmed A. Mohalhal ${ }^{1}$ - Dalia A. Ghalwash ${ }^{1} \cdot$ Ahmed A. Abdel-Kader $^{1}$.

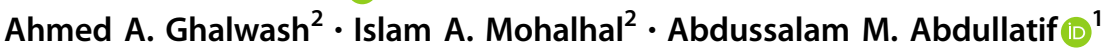

Received: 29 November 2018 / Accepted: 29 November 2018 / Published online: 19 December 2018

(c) The Royal College of Ophthalmologists 2018

We would like to thank Awasthi et al. [1] for their interest in our paper and their insightful comments.

Abdussalam M. Abdullatif

abdussalamabdullatif@kasralainy.edu.eg

1 Department of Ophthalmology, Kasr El Aini Hospital, Cairo University, El-Manial, Cairo, Egypt

2 Research Institute of Ophthalmology, Ministry of High Education, Giza, Egypt
Phacoemulsification with three-piece intraocular lens (IOL) implantation was done in all phakic patients as we believe that removing the lens gives access to the ora and far retinal periphery and allows dealing with the anterior proliferative vitreoretinopathy (PVR). The axial length was measured using optical biometry (IOL Master; Carl Zeiss, Oberkochen, Germany). If the axial length measured by optical biometry was shorter than that of the other eye, the axial length measurement was verified with A-scan ultrasonography [2]. SRK/T formula with the manufacturer's 